\title{
Cervical Radiculopathy: Incidence and Treatment of 1,420 Consecutive Cases
}

\author{
Han Jo Kim ${ }^{1}$, Venu M. Nemani ${ }^{2}$, Chaiwat Piyaskulkaew ${ }^{2}$, Samuel Romero Vargas ${ }^{2}$, K. Daniel Riew ${ }^{3}$ \\ ${ }^{1}$ Spine Service, Hospital for Special Surgery, Weill Cornell Medical College, New York, NY, USA \\ ${ }^{2}$ Department of Orthopaedic Surgery, Washington University School of Medicine, St. Louis, MO, USA \\ ${ }^{3}$ Spine Division, Department of Orthopaedic Surgery, Columbia University Medical Center, New York, NY, USA
}

\begin{abstract}
Study Design: Retrospective case series.
Purpose: To determine the incidence of cervical radiculopathy requiring operative intervention by level and to report on the methods of treatment.

Overview of Literature: Cervical radiculopathy is a common cause of pain and can result in progressive neurological deficits. Although the pathology is well understood, the actual incidence of cervical radiculopathy at particular spinal levels ultimately requiring operative intervention is unknown.

Methods: A large consecutive series of patients operated on by a single surgeon were retrospectively analyzed. The incidence of cervical radiculopathy at each level was defined for every patient. Procedures used for operative treatment were noted. Health related quality of life (HROL) scores were collected both pre-operatively and postoperatively.

Results: There were 1305 primary and 115 revision operations performed. The most common primary procedures performed were anterior cervical discectomy and fusion (ACDF, 50\%) and anterior cervical corpectomy and fusion (ACCF, 28\%). The most commonly affected levels were C6 (66\%) and C7 (62\%). Reasons for revision were pseudarthrosis (27\%), clinical adjacent segment pathology (CASP, $63 \%)$, persistent radiculopathy (11\%), and hardware-related (2.6\%). The most common procedures performed in the revision group were posterior cervical decompression and fusion (PCDF, $42 \%$ ) and ACDF (40\%). The most commonly affected levels were C7 (43\%) and $\mathrm{C5}(30 \%)$. Among patients that had their index surgery at our institution, the revision rate was $6.4 \%$. In both primary and revision cases there was a significant improvement in Neck Disability Index and visual analogue scale scores postoperatively. Postoperative HROL scores in the revision cases were significantly worse than those in the primary cases $(p<0.01)$.

Conclusions: This study provides the largest description of the incidence of cervical radiculopathy by level and operative outcomes in patients undergoing cervical decompression. The incidence of CASP was $4.2 \%$ in 3.3 years in this single institution series.
\end{abstract}

Keywords: Cervicalgia; Radiculopathy; Cervical; Cervical spondylosis; Spine osteoarthritis

\section{Introduction}

Cervical radiculopathy is a common cause of axial neck and arm pain, and is characterized by symptoms that radiate to the upper extremities in a dermatomal distribution. The sensory symptoms can also be accompanied by weakness or altered reflexes depending on the level(s) involved. Nerve root impingement can be secondary to

Received Jul 27, 2015; Revised Aug 17, 2015; Accepted Aug 20, 2015

Corresponding author: Han Jo Kim

Spine Service, Hospital for Special Surgery, 535 E 70th Street New York, NY 10021, USA

Tel: +1-212-774-2837, Fax: +1-646-797-8428, E-mail: hanjokimmd@gmail.com 
an acute disc herniation, or also from chronic spondylosis and/or instability with impingement from uncovertebral osteophytes, hypertrophied facet joints, and decreased disc height.

A population-based analysis in Rochester, Minnesota, reported an annual incidence of cervical radiculopathy of 107.3 per 100,000 for men and 63.5 per 100,000 for women with a peak at 50 to 54 years of age [1]. While most cases are self-limited, some are refractory to conservative care. When patients have failed non-operative management, a variety of procedures can be used to treat cervical radiculopathy depending on patient and surgeon factors. These include anterior cervical discectomy and fusion (ACDF), anterior cervical corpectomy and fusion (ACCF), posterior cervical decompression with or without fusion (PCDF), or cervical disc arthroplasty (CDA). In the Rochester series, $26 \%$ of the 561 patients underwent surgery within three months of the diagnosis [1].

What remains unknown, however, is the incidence of cervical radiculopathy at particular spinal levels that ultimately require operative intervention. This study examined, to our knowledge, the largest single-surgeon series of consecutive patients to define the incidence of cervical radiculopathy by level, and to report on the various methods for operative treatment.

\section{Materials and Methods}

Institutional Review Board approval was obtained prior to initiating this study. A consecutive series of patients operated on by a single surgeon from 2000 to 2010 for cervical radiculopathy were retrospectively analyzed. Cervical radiculopathy was defined as sensory symptoms (pain, numbness, or paresthesias) and/or motor symptoms attributed to compression of one or more cervical nerve roots. All patients had undergone and failed conservative management including (but not necessarily limited to) oral pain medications, immobilization, physical therapy, epidural or selective nerve root injections, or traction. Patients that had previously undergone cervical spine surgery for radiculopathy and needed revision surgery were included and separately analyzed.

For each patient, detailed demographic data was collected, and the incidence of cervical radiculopathy at each individual level was defined. Procedures used for operative treatment were also noted. Health related quality of life (HRQL) scores including Neck Disability Index
(NDI) and visual analogue scale (VAS) for neck and arm pain were collected both pre-operatively as well as postoperatively.

Overall summary statistics were calculated in terms of means and standard deviations for continuous variables and frequencies and percentages for discrete variables. Group differences for continuous variables between groups were calculated using independent samples $t$-test (or Mann-Whitney $U$ test if the assumption of normality was violated). Chi-squared or Fisher exact test were used for evaluating differences in categorical variables.

\section{Results}

\section{Primary cases}

There were 1,305 primary and 115 revision operations performed during the study period (Table 1). Of the primary procedures, 659 were men and 646 were female; 381 had previously undergone an operation at an outside institution and 924 had no previous cervical spine procedures. The most common levels affected in primary cases were C6 (66\%) and C7 (62\%), while the least commonly affected were C2 (1.5\%) and T1 (4.9\%). The distributions of levels affected were similar in both men and women (Table 2).

The number of affected and treated levels increased as age increased in primary cases. In patients undergoing primary surgery, patients $<40$ years of age had, on average, 1.53 levels operated on, while patients $>60$ years of

Table 1. Incidence of cervical radiculopathy by spinal level

\begin{tabular}{lcc} 
Surgical detail & Primaries & Revisions \\
No. of procedures & 1,305 & 115 \\
Levels & 2,811 & 198 \\
\hline Average levels/procedure & 2.2 & 1.7 \\
Levels affected & & \\
\hline C2 & $20(1.5)$ & 0 \\
\hline C3 & $96(7.4)$ & $8(7.0)$ \\
\hline C4 & $285(22)$ & $31(27)$ \\
C5 & $493(38)$ & $35(30)$ \\
\hline C6 & $855(66)$ & $31(27)$ \\
C7 & $803(62)$ & $49(43)$ \\
C8 & $195(15)$ & $33(29)$ \\
\hline T1 & $64(4.9)$ & $11(9.6)$ \\
\hline
\end{tabular}


Table 2. Involved spinal levels stratified by sex

\begin{tabular}{lcccccccc} 
Sex & C2 & C3 & C4 & C5 & C6 & C7 & C8 & T1 \\
Primaries & & & & & & & & \\
Male $(n=659)$ & 1.4 & 3.4 & 23.2 & 36.4 & 66.9 & 63.6 & 16.1 & 5.9 \\
\hline Female $(n=646)$ & 1.7 & 6.3 & 20.4 & 39.2 & 64.1 & 59.4 & 13.8 & 3.9 \\
\hline Revisions & & & & & & & & \\
\hline Male $(n=49)$ & 0 & 6.1 & 30.6 & 34.7 & 36.7 & 46.9 & 38.8 & 14.3 \\
\hline Female $(n=66)$ & 0 & 7.6 & 24.2 & 27.3 & 19.7 & 39.4 & 21.2 & 6.1 \\
\hline
\end{tabular}

Table 3. Involved spinal levels stratified by patient age and revision status

\begin{tabular}{lcccccccc} 
Age (yr) & C2 & C3 & C4 & C5 & C6 & C7 & C8 & T1 \\
Primaries & & & & & & & & \\
\hline$<0(n=163)$ & 0 & 3.1 & 8.0 & 16.6 & 61.3 & 58.3 & 5.5 & 0 \\
\hline $40-60(n=957)$ & 1.3 & 6.5 & 21.9 & 39.4 & 66.0 & 62.9 & 13.7 & 4.8 \\
\hline$>60(n=185)$ & 4.3 & 15.7 & 33.5 & 48.1 & 66.5 & 57.3 & 29.7 & 9.7 \\
\hline Revisions & 0 & 0 & 16.7 & 66.7 & 50.0 & 33.3 & 0 & 0 \\
\hline $40(n=6)$ & 0 & 6.7 & 27.8 & 30.0 & 25.6 & 43.3 & 27.8 & 10.0 \\
\hline $40-60(n=90)$ & 0 & 10.5 & 26.3 & 21.1 & 26.3 & 42.1 & 42.1 & 10.5 \\
\hline$>60(n=19)$ & & & & & & & \\
\hline
\end{tabular}

Percentages reflect involvement of a particular level with respect to the total number of patients. And percentages add up to greater than $100 \%$ because multiple levels were often involved in the same patient.

age had, on average, 2.65 levels operated on $\left(p=1.4 \times 10^{-15}\right)$ (Table 3). C6 and C7 were the levels most commonly operated on.

The most common procedure performed among the primary group was ACDF (50\%) (Table 4). Less commonly performed were ACCF (28\%), posterior cervical decompression $+/$ - fusion (PCDF, 19\%), and anterior cervical discectomy and arthroplasty (ACDA, 3.1\%). Patients undergoing primary surgery who had posterior-only procedures had an average of 2.6 levels operated on compared to 2.0 levels in patients undergoing anterior or anteriorposterior procedures $\left(p=6.9 \times 10^{-10}\right)$. Patients who received ACDA were on average younger than patients who had ACDF, ACCF, or a posterior procedure (44.9 years ACDA vs. 51.3 years other procedures, $p=0.002$ ).

Considering only the 381 patients that had previous cervical spine surgery elsewhere but were having their first procedure performed at our institution, the most commonly affected levels were similarly C7 (58\%) and C6 (45\%). The most common procedure performed in this cohort of patients was also ACDF (42\%). A higher percentage of these patients had PCDF (37\%) reflecting the fact that these patients had already had a previous surgery (almost universally an ACDF) done elsewhere. Thirtynine of these patients had to have a subsequent revision procedure after their first procedure done at our institution for an overall revision rate of $10.2 \%$.

\section{Revision cases}

Ninety-eight patients underwent 115 revision procedures, with nine patients having multiple revision procedures (Table 1). There was no difference in the number of levels operated on when comparing patients $<40$ years of age (1.67 levels) and those $>60$ years of age (1.79 levels) ( $p=0.87$ ) (Table 3). In the revision setting, C5 was the level most frequently operated on in patients $<40$ years of age, while C6 and C7 were the most frequently levels operated on in those patients $>40$ years of age. Reasons for revision were clinical adjacent segment pathology (CASP, 63\%), pseudarthrosis (27\%), persistent radiculopathy (11\%), and hardware-related (2.6\%). CASP was equally split, affecting either more cephalad levels or more caudad levels (50\% each). The most common procedure performed in the re- 
Table 4. Operations performed for cervical radiculopathy $(n=1,695)$

\begin{tabular}{lcc} 
Procedures & $\begin{array}{c}\text { Primaries } \\
(\mathrm{n}=1,567)\end{array}$ & $\begin{array}{c}\text { Revisions } \\
(\mathrm{n}=128)\end{array}$ \\
ACDF & $786(50)$ & $51(40)$ \\
ACCF & $439(28)$ & $17(13)$ \\
PCDF & $287(19)$ & $54(42)$ \\
ACDA & $49(3.1)$ & $6(4.7)$ \\
\hline
\end{tabular}

Values are presented as number of level (\%).

ACDF, anterior cervical discectomy and fusion; ACCF, anterior cervical corpectomy and fusion; PCDF, posterior cervical decompression +/fusion; ACDA, anterior cervical disc arthroplasty.

Table 5. Revision rate stratified by procedure among patients operated on for cervical radiculopathy at a single institution

\begin{tabular}{lc} 
Procedure & Revision rate (\%) \\
ACDF & 4.2 \\
ACCF & 8.3 \\
PCD & 11.6 \\
PCDF & 9.4 \\
ACDA & 14.3 \\
\hline
\end{tabular}

ACDF, anterior cervical discectomy and fusion; ACCF, anterior cervical corpectomy and fusion; PCD, posterior cervical decompression; PCDF, posterior cervical decompression and fusion; ACDA, anterior cervical disc arthroplasty.

Table 6. HROL scores in patients operated on for cervical radiculopathy

\begin{tabular}{lcc} 
& Primaries & Revisions \\
Pre-op HROL & & \\
\hline VAS neck & 6.5 & 6.4 \\
VAS arm & 6.2 & 6.0 \\
NDI & 21.9 & 23.1 \\
Post-op HROL & & \\
\hline VAS neck & 3.9 & 4.8 \\
\hline VAS arm & 3.3 & 4.4 \\
NDI & 15.8 & 19.5 \\
\hline
\end{tabular}

HROL, health related quality of life; Pre-op, preoperatively; VAS, visual analogue scale; NDI, Neck Disability Index; Post-op, postoperatively.

vision group was PCDF (42\%), followed by ACDF (40\%), ACCF (13\%), and ACDA (4.7\%) (Table 4). The most commonly affected spinal levels were C7 (43\%) and C5 (30\%), while the least commonly affected were C2 (0\%) and C3 (7.0\%).

Notably, there were more posterior procedures done in the revision setting compared to primary procedures.
However, the approach varied significantly depending on the indication for revision surgery. In cases performed for pseudarthrosis, PCDF was performed at $75 \%$ of the affected levels compared to ACDF at $22 \%$ of the affected levels. In contrast, in cases performed for adjacent segment disease, ACDF was performed at $52 \%$ of the affected levels compared to PCDF at $29 \%$ of affected levels.

Considering only the 924 patients that had primary surgery at our institution, 59 of these patients had 66 revision procedures for an overall revision rate of $6.4 \%$. Revisions were performed in $4.2 \%$ for CASP and $1.7 \%$ for pseudarthrosis (at 3.3-year average follow-up). Of these revision procedures, $40 \%$ overall were ACDF and 39\% were PCDF. Again, however, the procedures performed were markedly different depending on the reason for revision. For pseudarthrosis, $93 \%$ of procedures were PCDF. For a diagnosis of CASP, however, only $23 \%$ had PCDF and the remainder had an anterior procedure (56\% ACDF, 12\% ACCF, and 9\% ACDA). Further, the revision rate varied significantly depending on the primary procedure $(p=0.02)$ (Table 5), with a relative risk of 2.8 of needing a revision after a PCD compared to ACDF. There was no increase in revision rate for patients that were smokers $(8.2 \%$ smokers vs. $5.5 \%$ non-smokers, $p=0.12)$ or patients that were diabetic $(7.1 \%$ diabetic vs. $6.3 \%$ non-diabetic, $p=0.81$ ).

In both primary and revision cases there was a significant improvement in NDI, VAS neck, and VAS arm scores postoperatively compared to pre-operatively. However, the postoperative HRQL scores in the revision cases were significantly worse than those in the primary cases $(p<0.01)$ (Table 6).

\section{Discussion}

Cervical radiculopathy can be a disabling disorder causing upper extremity pain and weakness. While most cases are self-limited and resolve with conservative treatment [1,2], some eventually require operative intervention. Here we present the largest reported series of patients to date who were operated on for a diagnosis of cervical radiculopathy by a single surgeon (K.D.R.) at a single institution. The levels most often affected were C6 and C7. The most common primary procedure performed to treat radiculopathy was ACDF (50\% of cases). However, in revision settings a posterior decompression and fusion was utilized more commonly, especially in cases of pseudarthrosis after a previous anterior procedure. In this large single-institu- 
tion series, the overall revision rate was $6.4 \%$ for cases where the index procedure was performed at the same institution. CASP was the most common reason for revision surgery comprising $4.2 \%$ of cases at 3.3-year followup. Revisions for pseudarthrosis were performed in $1.7 \%$ of the cases.

\section{Levels affected}

In our series of patients who underwent operative treatment of cervical radiculopathy, C6 and C7 were the most commonly affected levels. This is consistent with the findings of several previous studies showing that the C5-6 and C6-7 disc levels are the most susceptible to degenerative disease and resulting nerve root impingement [3-5]. In a small series of 40 surgical patients, C6 and C7 were the most frequently involved levels [6]. These studies are also consistent with biomechanical work examining the kinematics of the cervical spine, which demonstrated that C5-6 and C6-7 are the levels of maximum flexion and extension and subject to high loads $[7,8]$. Interestingly, there is also data showing anatomic variability between spinal levels that may account for the higher incidence of symptomatic radiculopathy at C5-6 and C6-7. Cadaveric dissections in the lower cervical spine revealed that at the C5-6 and C6-7 levels the intervertebral disc has an "axillary" relationship to the nerve root, which may have an increased propensity for symptomatic compression compared to the C7-T1 disc, which usually had no direct contact with the $\mathrm{C} 8$ nerve root [9]. Consistent with this, there was a much lower incidence of $\mathrm{C} 8$ radiculopathy needing surgery compared to C5-7 in our patient series (Table 1).

\section{Choice of procedure}

There is no evidence for a single superior surgical approach and/or technique to treat cervical radiculopathy. ACDF has traditionally been used with great success for the treatment of cervical radiculopathy from spondylosis [10]. An anterior approach allows for restoration of cervical lordosis and spinal stabilization through fusion, and allows easy access to anterior compressive lesions, such as central or paracentral disc herniations or uncovertebral osteophytes, without the need for neural retraction. ACDA has also been shown by several groups to be a successful motion-preserving technique for the treatment of cervical radiculopathy [11]. An anterior foraminotomy has also been described, however, the long-term results for this procedure have been controversial and so it was rarely utilized in our series. A posterior laminoforaminotomy, similarly avoids the potential complications associated with fusion, such as pseudarthrosis, and to some extent adjacent segment degeneration. This can also be done through minimally invasive techniques or traditional open techniques with good results $[12,13]$. Instrumented fusion via a posterior approach can also be performed with the use of segmental screw fixation.

In this study, $50 \%$ of the primary procedures performed for radiculopathy were ACDFs, with $81 \%$ of all procedures being done via an anterior approach (ACDF, ACCF, and ACDA). In our practice, we have reliably had success using these techniques as this approach allows central and foraminal decompression, as well as indirect foraminal decompression via distraction with an interbody graft or implant in addition to direct uncinatetecomy when necessary. However, we performed the latter only in cases with neurologic deficits associated with severe foraminal stenosis due to uncinate hypertrophy given the increased risk of vertebral artery injury.

In contrast, a posterior decompression and fusion was used much more frequently in the revision setting, especially in cases of pseudarthrosis. If there is cervical radiculopathy with minimal central stenosis or significant spondylosis, a posterior foraminotomy is also a good option providing the benefit of directly visualizing exposing, and decompressing the nerve root without needing to proceed with fusion. Ideal patients for an initial posterior decompression are those with soft lateral disc herniations, or those that are at increased risk for complications with a fusion. In our experience, patients who would benefit from a posterior foraminotomy will have a positive Spurlings maneuver and improvement with forward flexion, neck rotation to the contralateral side with arm elevation. Ultimately, the procedure chosen should be one that the surgeon is comfortable performing, addresses the relevant pathophysiology causing neural compression, and provides consistent and reproducible results. Our results in performing mostly anteriorly-based procedures in primary cases represent our institutional bias. When an anteriorly-based procedure failed secondary to pseudarthrosis, the decision was made to go to a posterior procedure to increase stability without having to remove the anterior instrumentation and to avoid operating through scarred tissue. However in cases of CASP, we usually performed 
an anteriorly-based procedure.

\section{Revision surgery}

The overall revision rate among patients who had their primary operation at our institution was $6.4 \%$, with $4.2 \%$ being for CASP at an average 3.3-year follow-up. The overall revision rate in this series is less than that previously reported for cervical spine surgery for radiculopathy or myelopathy. van Eck et al. [14] recently reported an overall revision rate of $15 \%$ in patients who underwent ACDF for radiculopathy or myelopathy compared to a $4.2 \%$ revision rate in our series of patients who underwent ACDF (Table 5). In the same paper, they reported a revision rate of 7\% for CASP at 2.6 years follow-up [14] compared to $4.2 \%$ at 3.3 years in our series. The reason for the difference in revision rates are unclear as the techniques used were likely similar as the patients were operated on during the same time period (2000-2010) and all had supplemental plate fixation.

Interestingly, however, we found higher revision rates among patients who underwent PCD without fusion and those who underwent CDA compared to those that underwent ACDF (Table 5). This is consistent with some previous reports of higher reoperation rates in patients undergoing PCD. Bydon et al. [15] recently reported a $9.9 \%$ reoperation rate after posterior foraminotomy at an average of 2.4 years after the index operation. The reoperation rate increased to $18.3 \%$ in those patients with follow-up periods greater than 2 years [15]. However, Jagannathan et al. [16] reported that at approximately 6.5 years of follow-up only $3.1 \%$ of patients required revision cervical spine surgery after initial posterior foraminotomy. Certainly, not fusing the affected level leaves open the possibility of recurrent disc herniations or regeneration of osteophytes that can cause neural impingement. However, in certain carefully selected patients, ACDA or PCD can provide good symptomatic relief with a low revision rate.

One of the major benefits of this study is that it represents the results of a single surgeon at a single institution, thus eliminating systematic factors that could confound the data. For the same reason, however, it does not preclude the possibility that the surgeon favors one particular type of procedure over another, thus affecting the results when comparing different operations. However, the senior author has significant experience with all of the procedures performed; thus, we do not believe this affects the results. A limitation of the study is that we do not have long-term follow-up, with an average follow-up of only 3.3 years. However, for a study concerning the levels operated on, follow-up is less relevant.

\section{Conclusions}

This study provides the first description of the incidence of cervical radiculopathy by level and operative outcomes in a large series of patients undergoing cervical decompression by a single surgeon at a single institution. The most common levels requiring operative intervention were $\mathrm{C} 6$ and $\mathrm{C} 7$. The incidence of CASP requiring revision surgery was $4.2 \%$ at 3.3 years in this single institution series, with an overall revision rate of $6.4 \%$. Patients undergoing ACDF had the lowest revision rate at $4.2 \%$.

\section{Conflict of Interest}

No potential conflict of interest relevant to this article was reported.

\section{Acknowledgments}

Research Performed by the Cervical Spine Service, Washington University Orthopedics, Washington University in St. Louis, MO, USA

\section{References}

1. Radhakrishnan K, Litchy WJ, O'Fallon WM, Kurland LT. Epidemiology of cervical radiculopathy: a population-based study from Rochester, Minnesota, 1976 through 1990. Brain 1994;117(Pt 2):325-35.

2. Sampath P, Bendebba M, Davis JD, Ducker T. Outcome in patients with cervical radiculopathy: prospective, multicenter study with independent clinical review. Spine (Phila Pa 1976) 1999;24:591-7.

3. Fager CA. Management of cervical disc lesions and spondylosis by posterior approaches. Clin Neurosurg 1977;24:488-507.

4. Friedenberg ZB, Miller WT. Degenerative disc disease of the cervical spine. J Bone Joint Surg Am 1963;45:1171-8.

5. Murphey F, Simmons JC, Brunson B. Chapter 2. ruptured cervical discs, 1939 to 1972. Clin Neurosurg 1973;20:9-17. 
6. Kelsey JL, Githens PB, Walter SD, et al. An epidemiological study of acute prolapsed cervical intervertebral disc. J Bone Joint Surg Am 1984;66:907-14.

7. Lysell E. Motion in the cervical spine: an experimental study on autopsy specimens. Acta Orthop Scand 1969:Suppl 123:1+.

8. White AA 3rd, Panjabi MM. The basic kinematics of the human spine: a review of past and current knowledge. Spine (Phila Pa 1976) 1978;3:12-20.

9. Tanaka N, Fujimoto Y, An HS, Ikuta Y, Yasuda M. The anatomic relation among the nerve roots, intervertebral foramina, and intervertebral discs of the cervical spine. Spine (Phila Pa 1976) 2000;25:286-91.

10. Bohlman HH, Emery SE, Goodfellow DB, Jones PK. Robinson anterior cervical discectomy and arthrodesis for cervical radiculopathy: long-term follow-up of one hundred and twenty-two patients. J Bone Joint Surg Am 1993;75:1298-307.

11. Sasso RC, Anderson PA, Riew KD, Heller JG. Results of cervical arthroplasty compared with anterior discectomy and fusion: four-year clinical outcomes in a prospective, randomized controlled trial. J Bone Joint Surg Am 2011;93:1684-92.

12. Henderson CM, Hennessy RG, Shuey HM Jr, Shack- elford EG. Posterior-lateral foraminotomy as an exclusive operative technique for cervical radiculopathy: a review of 846 consecutively operated cases. Neurosurgery 1983;13:504-12.

13. Heary RF, Ryken TC, Matz PG, et al. Cervical laminoforaminotomy for the treatment of cervical degenerative radiculopathy. J Neurosurg Spine 2009;11: 198-202.

14. van Eck CF, Regan C, Donaldson WF, Kang JD, Lee JY. The revision rate and occurrence of adjacent segment disease after anterior cervical discectomy and fusion: a study of 672 consecutive patients. Spine (Phila Pa 1976) 2014;39:2143-7.

15. Bydon M, Mathios D, Macki M, et al. Long-term patient outcomes after posterior cervical foraminotomy: an analysis of 151 cases. J Neurosurg Spine 2014; 21:727-31.

16. Jagannathan J, Sherman JH, Szabo T, Shaffrey CI, Jane JA. The posterior cervical foraminotomy in the treatment of cervical disc/osteophyte disease: a single-surgeon experience with a minimum of 5 years' clinical and radiographic follow-up. J Neurosurg Spine 2009;10:347-56. 